\title{
Retrospective Comparative Study between Open and Modified Ultrasound-guided Percutaneous Achilles Tendon Repair
}

\author{
Keith Hay-Man Wan ${ }^{1}$, Michael Siu-Hei Tse ${ }^{2}$, Irene Oi-Lam Lo ${ }^{3}$, Simon Chi-Pan Yuen ${ }^{4}$, Richard Hin-Lun Lee ${ }^{5}$, Kam-Kwong Wong ${ }^{6}$
}

\begin{abstract}
Introduction: To compare the outcomes between the percutaneous repair technique of the Achilles tendon rupture with the open repair technique.

Materials and methods: A retrospective review of patients with complete Achilles tendon rupture managed surgically in our institution from January 2015 to June 2019. Group I consisted of patients managed with the percutaneous repair technique. Group II consisted of patients managed with the open technique. Clinical outcomes including re-rupture, wound infection, and pain control were compared.

Results: Fifty-two patients were included in the study with 22 patients allocated into group I and 30 patients allocated to group II. The mean age of group I was 41.2 (range 21-78, SD 16.097). The mean follow-up time was 38.4 months (range 14-55 months, SDV 12.738). The mean age of group II was 48.8 (range 27-80, SDV 14.938). At postoperative 1 year, there was no statistical difference in the range of motion. Among the patients in group I, the mean numeral pain rating scale (NPRS) was statistically lower than that of the patients in group II. There was no wound infection, sural nerve injury, or re-rupture in group I. Two patients in group II developed postoperative wound infection. One of them had been complicated with the re-rupture of the repaired Achilles tendon.

Conclusion: The percutaneous repair technique is a reasonable treatment option for ruptured Achilles tendon, with a satisfactory medium-term outcome comparable, if not better, than the traditional open repair technique.

Keywords: Achilles tendon repair, Foot and ankle surgery, Minimal invasive, Surgical technique.

Journal of Foot and Ankle Surgery (Asia Pacific) (2022): 10.5005/jp-journals-10040-1178
\end{abstract}

\section{INTRODUCTION}

Achilles tendon rupture is one of the most common injuries in the foot and ankle region. Although it is a common sporting injury affecting patients in their relative "prime" age for sports between 30 and 50, ${ }^{1,2}$ considerable controversies still exist over the management options. It is well accepted that open surgical repair is associated with a lower risk of re-rupture, ranging from 1.4 to $2.8 \%,{ }^{1,3}$ as opposed to the higher risk of re-rupture with conservative management. ${ }^{4}$ However, the downside is a higher risk of complications including wound infection and necrosis. ${ }^{4,5}$

Percutaneous repair of the Achilles tendon, first described by $\mathrm{Ma}$ and Griffith, ${ }^{6}$ offers a minimally invasive solution to repair the tendon. Several studies have shown that a modified version of the technique described by the original authors had significantly increased the biomechanical strength of the repair, 7,8 with satisfactory functional outcome. ${ }^{9,10}$ Though not frequently encountered, sural nerve injury is an important complication of the percutaneous method. We have therefore introduced the use of intraoperative ultrasonography to lower the risk of sural nerve injury.

The purpose of this study was to compare the outcomes of this modified percutaneous repair technique with that of the traditional open repair of the Achilles tendon. We hypothesized that the percutaneous technique would achieve a comparable outcome, with fewer wound complications.

\section{Materials and Methods}

A retrospective analysis of patients with complete rupture of Achilles tendon managed surgically from January 2015 to June 2019 in our institution. The inclusion criteria were (1) a closed

\begin{abstract}
${ }^{1-6}$ Department of Orthopaedics and Traumatology, Kwong Wah Hospital, Hong Kong

Corresponding Author: Keith Hay-Man Wan, Department of Orthopaedics and Traumatology, Kwong Wah Hospital, Hong Kong, Phone: +852 35172776, e-mail: keithayman@hotmail.com

How to cite this article:Wan KH-M, Tse MS-H, Lo IO-L, et al. Retrospective Comparative Study between Open and Modified Ultrasound-guided Percutaneous Achilles Tendon Repair. J Foot Ankle Surg (Asia Pacific) 2022;9(1):7-11.
\end{abstract}

Source of support: Nil

Conflict of interest: None

complete Achilles tendon rupture; (2) an acute injury that occurred $<7$ days before the surgery; (3) a rupture over the tendinous part of the tendon with at least $3 \mathrm{~cm}$ distal stump; and (4) patients who provided informed consent.

Exclusion criteria included (1) previous surgical procedures or history of rupture of the ipsilateral Achilles tendon; (2) history of local injection or topical medication which may have weakened the tendon; (3) mental incapacitation and major medical comorbidities such as cerebrovascular accidents which may affect patient's ability to comply to a standardized rehabilitation regime.

The patients were divided into two cohorts. Group I consisted of patients managed with the percutaneous repair technique. Group II consisted of patients managed with the traditional open technique.

The diagnosis of Achilles tendon rupture was based on the following criteria: (1) clinically palpable gap; (2) positive Thompson test; ${ }^{11}$ and (3) confirmation by intraoperative ultrasonography.

() The Author(s). 2022 Open Access This article is distributed under the terms of the Creative Commons Attribution 4.0 International License (https://creativecommons. org/licenses/by-nc/4.0/), which permits unrestricted use, distribution, and non-commercial reproduction in any medium, provided you give appropriate credit to the original author(s) and the source, provide a link to the Creative Commons license, and indicate if changes were made. The Creative Commons Public Domain Dedication waiver (http://creativecommons.org/publicdomain/zero/1.0/) applies to the data made available in this article, unless otherwise stated. 


\section{Surgical Procedure}

\section{Percutaneous Repair Technique}

The procedure was performed with the patient in a prone position without a thigh tourniquet. No prophylactic antibiotic or antithrombotic was given. The ankle was put into $25^{\circ}$ of plantar flexion by a folded towel. Ultrasound examination was performed at the theater to identify the torn site of the Achilles tendon and to document the length of the distal stump. In addition, the location of the sural nerve was identified with the long axis of the probe placed over the lateral side of the calf. A cadaveric study performed by Citak et al. ${ }^{12}$ revealed that the lateral crossing of the sural nerve was $8.7-12.4 \mathrm{~cm}$ proximal to the calcaneal tuberosity, so that the pin should not pass through the substance of the Achilles tendon $9 \mathrm{~cm}$ proximal to the calcaneal tuberosity.

The Achilles tendon was then repaired using the modified technique, aiming at a cross-configuration of sutures at both the proximal and distal stumps. A heavy-duty, braided, and nonabsorbable suture was used (FiberWire-Arthrex). Figure 1 shows the individual numbering of the percutaneous incisions. Firstly, a suture on a beath guide pin was passed transversely through the tendon (from incision 1 to 2 ), followed by a cross and diagonal suture (from incision 2 to 3). A second pin with the suture was passed (from incision 1 to 4). Two needles were used simultaneously, with the first pin placed in the tendon while the second pin was passed to avoid cutting the suture during the crossing (Fig. 2). During each puncture with the guide pin, the ultrasound probe was placed over the long axis of the tendon to ensure adequate placement of the pin through the substance of the tendon. Next, the pin was brought proximally along the subcutaneous planes (from incision 4 to 8 ; from incision 3 to 7). The crossing was repeated similarly (from incision 7 to 6 ; incision 8 to 5) (Fig. 3). After that, the sutures were brought extra-tendinously back distally (from incision 5 to 3; incision 6 to 4). After approximating the torn Achilles tendon, the lateral end of the suture was passed medially (from incision 4 to 3 ), the suture is tied and the knots were buried subcutaneously. Altogether only eight small stab incisions were made.

After the repair, the approximation of the Achilles tendon was verified by ultrasonography. Intraoperative Thompson's test was performed to look for plantar flexion of the foot upon squeezing the calf.

Postoperative rehabilitation consisted of cast immobilization in $20^{\circ}$ of plantar flexion and non-weight bearing walking for 3 weeks, followed by heel-raise shoes and partial weight-bearing walking for 3 weeks.

\section{Open Technique}

The procedure was performed in a prone position with a thigh tourniquet. No prophylactic antibiotic or antithrombotic was given. Ultrasound examination was performed at the theater to identify the torn site of the Achilles tendon and to document the length of the distal stump. A paramedian midline incision was made. Repair of the Achilles tendon was done with a non-absorbable suture (FiberWire-Arthrex) using a Krakow configuration.

Postoperative rehabilitation consisted of initial cast immobilization and non-weight bearing walking for 3 weeks, followed by ankle brace in plantar flexion for 3 weeks. Partial weight-bearing walking was started from postoperative 6 weeks. The patient was allowed to take off the brace with full weightbearing walking from postoperative 9 weeks.

All patients were followed up regularly at 3 weeks and 6 weeks postoperatively, then 3 months, 6 months, and 1 year.

The outcome measures were functional outcomes including the range of motion of the ankle, pain, and postoperative complications including wound infection and tendon re-rupture.

Statistical analysis was performed using SPSS (Version 26.0). An unpaired $t$-test was used to compare the demographics of the two groups. All continuous data were expressed in terms of mean and standard deviation (SD). The Fisher's exact test was used to analyze the categorical outcome of complications between the two groups. A $p$ value of $<0.05$ was taken as statistically significant.

\section{RESULTS}

A total of 55 patients were recruited. Three patients were excluded from the study as they had defaulted follow-up within the first postoperative year. Fifty-two patients were included (22 patients in group I and 30 patients in group II).

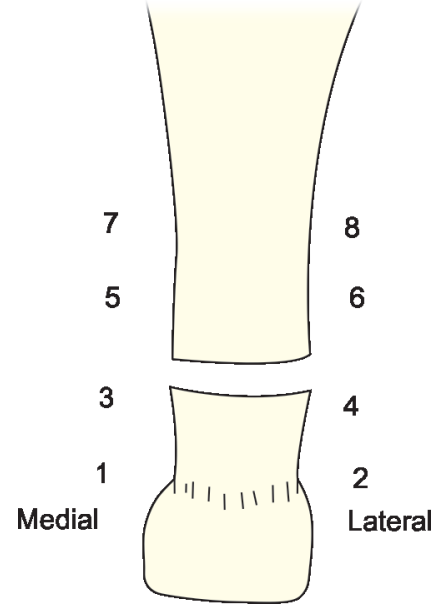

Fig. 1: Schematic diagram showing the numbering of the eight percutaneous incisions

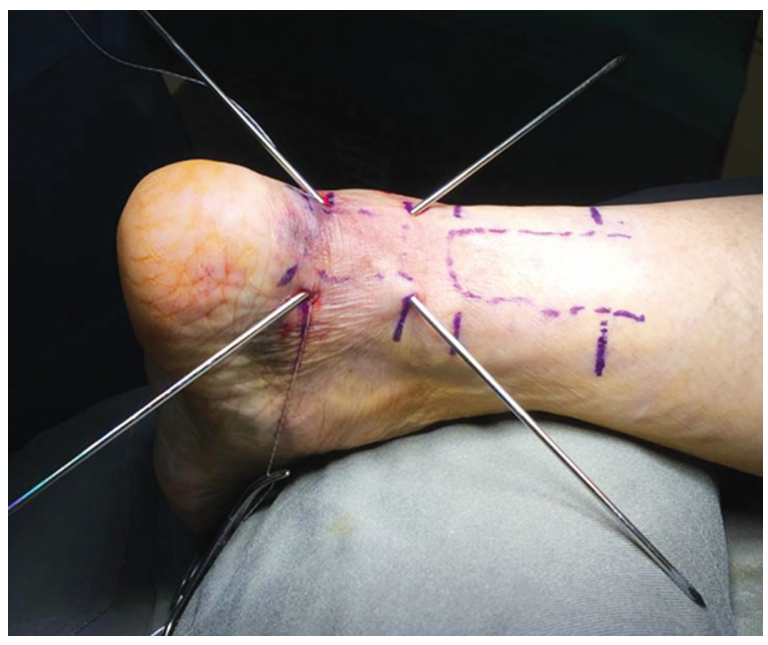

Fig. 2: The second pin was passed from incision 1 to 4 . At the same time, the first pin from incision 2 to 3 was kept in the distal stump of the tendon to protect the suture from being cut by the incoming second pin 
Group I consisted of 22 patients with a mean age of 41.2 (range 21-78, SD 16.097). The mean follow-up time was 38.4 months (range 14-55 months, SDV 12.738). Group II consisted of 30 patients with a mean age of 48.8 (range $27-80$, SDV 14.938). There was no statistical difference between the two groups in terms of demographic and follow-up time (Table 1).

At postoperative 1 year, there was no statistical difference in the range of motion of the ankle. Among the patients in group I the mean numeral pain rating scale (NPRS) was statistically lower than that of the patients in group II (Table 2). There was no statistical difference in the postoperative ankle plantar flexion power.

There was no wound infection, sural nerve injury, or re-rupture in group I. Two patients in group II were complicated with wound infection within 3 months postoperation. Of which one patient was managed successfully with an intravenous antibiotic. Another patient required surgical debridement. Intraoperatively, the Achilles tendon was found to be re-ruptured. The infection was finally eradicated but the patient decided against surgery to repair the torn Achilles tendon.

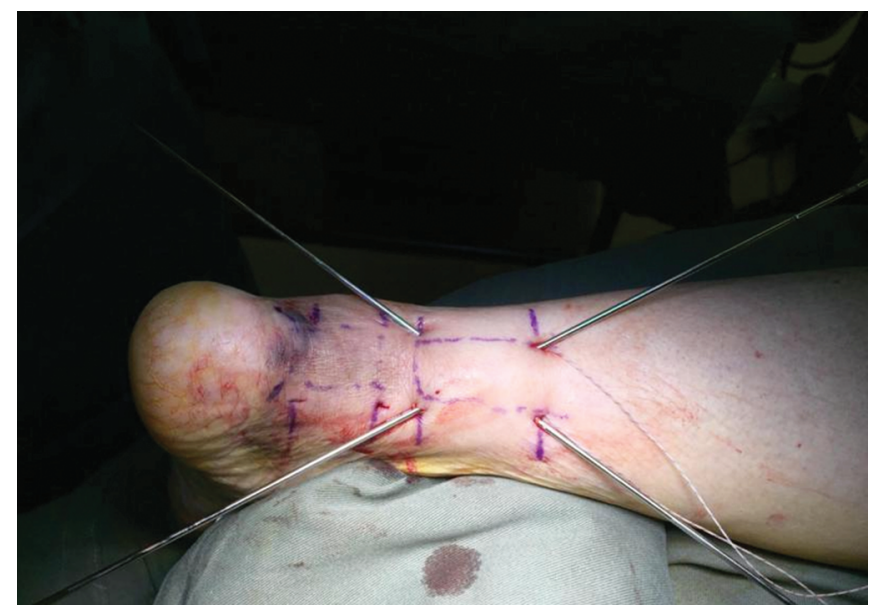

Fig. 3: Pin from incision 7 to 6, another pin from incision 8 to 5 . Again two pins were kept inside the tendon to avoid cutting of the sutures

\section{Discussion}

The ideal management option, let alone the ideal surgical technique for the treatment of Achilles tendon rupture, still remains controversial. Open surgical repair is the traditional surgical option that allows surgeons to directly visualize the tendon and the quality of the repair. In addition, the sural nerve can be identified and protected. However, when compared with conservative management, open surgical repair has shown a significantly higher rate of morbidity, including wound dehiscence, infection, and necrosis. ${ }^{3,13}$ First reported by Ma and Griffith's in the 1970s, percutaneous Achilles tendon repair offers a minimally invasive option with minimal surgical dissection.

The para-Tenon is a highly vascular structure that provides an important blood supply to the Achilles tendon. ${ }^{14}$ The percutaneous method allows for minimal dissection of the para-Tenon and the rupture site, and hence help to preserve the already scarce blood supply in the mid-portion of the Achilles tendon. ${ }^{15}$

The percutaneous method has previously been put into doubt. Some people have criticized it to be a blind method without clear visualization of the tear site and the quality of the repair, ${ }^{13}$ and the risk of injuring the sural nerve. ${ }^{16}$ We modified the surgical method proposed by Cretnik et al. ${ }^{9}$ with the incorporation of intraoperative ultrasonography. The location of the sural nerve was identified before the incision by placing the long axis of the probe along the lateral side of the Achilles tendon. The sural nerve was hence protected during the subsequent passage of the beath pin. During each passage of the pin, the ultrasound probe was placed along the long axis of the Achilles tendon to ensure that that the placement of the pin was neither too superficial nor too deep. This ensured a maximal repair strength and avoided the cutout of the sutures by the suboptimal placement of the sutures.

Our series of cases demonstrated satisfactory outcomes in terms of ankle range of motion, pain scores, which are comparable to the results in other series using the percutaneous technique. ${ }^{9,13}$ Compared with our group of patients with open repair, there was no statistical difference in the postoperative range of motion and re-rupture rate. Though not statistically

Table 1: Demographic in groups I and II

\begin{tabular}{|c|c|c|c|c|c|c|c|}
\hline & \multicolumn{3}{|c|}{ Group I } & \multicolumn{3}{|c|}{ Group II } & \multirow[b]{2}{*}{$p$ value } \\
\hline & Mean & $S D$ & Range & Mean & $S D$ & Range & \\
\hline Age & 41.2 & 16.097 & $21-78$ & 48.8 & 14.938 & $27-80$ & 0.0861 \\
\hline Male/female & & $18: 4$ & & & $25: 5$ & & \\
\hline FU (months) & 38.4 & 12.738 & $14-55$ & 40.9 & 17.204 & $13-66$ & 0.427 \\
\hline
\end{tabular}

Table 2: Comparison of the postoperative outcomes between groups I and II

\begin{tabular}{|c|c|c|c|c|c|c|c|}
\hline & \multicolumn{3}{|c|}{ Group I } & \multicolumn{3}{|c|}{ Group II } & \multirow[b]{2}{*}{$p$ value } \\
\hline & Mean & $S D$ & Range & Mean & $S D$ & Range & \\
\hline $\mathrm{DF}\left({ }^{\circ}\right)$ & 18.8 & 4.160 & $10-25$ & 17.7 & 4.568 & $10-25$ & 0.2774 \\
\hline $\mathrm{PF}\left({ }^{\circ}\right)$ & 44.6 & 9.233 & $30-60$ & 38.7 & 8.307 & $20-50$ & 0.0676 \\
\hline NPRS & 0.846 & 0.801 & $0-2$ & 1.684 & 1.108 & $0-3$ & 0.0264 \\
\hline \multirow[t]{2}{*}{ Ankle PF power } & 4.85 & 0.376 & $4-5$ & 4.84 & 0.376 & $3-5$ & 0.9763 \\
\hline & & Group I & & Group II & & $p$ value & \\
\hline Wound infection & & $0 / 22$ & & $2 / 30$ & & 0.502 & \\
\hline Re-rupture & & $0 / 22$ & & $1 / 30$ & & 1.000 & \\
\hline Sural nerve injury & & $0 / 22$ & & $1 / 30$ & & 1.000 & \\
\hline
\end{tabular}


significant, the wound infection rate was also lower for the percutaneous repair group. Postoperative pain control was statistically lower for the percutaneous repair group in terms of NPRS.

The reduced wound complication and superior pain control for the percutaneous repair could be explained by the approach of using stab incisions, as opposed to a long longitudinal incision in the open repair group. The percutaneous method had the advantage of minimal disruption of the para-Tenon, which is of paramount importance to the vascular supply and subsequent healing of the Achilles tendon. In contrast, the para-Tenon was often incised to approach the Achilles tendon during the open repair.

The major limitation of the study was that it had a retrospective design with a lack of randomization. Whether the patients were allocated to the percutaneous repair group and open repair group was based on the individual surgeon's preference.

Another limitation was that the cases were done by different surgeons in our institution. Although this may result in the lack of homogeneity in the surgical team, we believe that a good surgical technique can be readily reproduced by different surgeons safely and uniformly, rather than one which requires highly individualized expertise.

In addition, with regards to postoperative calf power, we were only able to provide such assessment using the Medical Research Council (MRC) scale for muscle strength. ${ }^{17}$ An important objective assessment of the Achilles tendon strength is isokinetic testing, ${ }^{13}$ but this was not available in our study due to financial and logistical constraints.

Another major limitation of this series was the small sample size. A strict inclusion criterion of a minimum of 1-year postoperative follow-up was adhered to in order to investigate the medium-term outcome of the percutaneous repair. A prospective randomized controlled with large sample size and longer follow-up duration would be the ideal next step to further evaluate the clinical outcome of the percutaneous repair technique.

We were aware of many other minimally invasive techniques for repairing the Achilles tendon in the literature. By no means, we were saying that our percutaneous repair technique was superior to other techniques. We aimed to demonstrate that our technique was a reasonable option with a satisfactory clinical outcome.

\section{ConcLusion}

The percutaneous repair technique is a reasonable treatment option for ruptured Achilles tendon, with a satisfactory mediumterm outcome comparable, if not better, than the traditional open repair technique.

\section{References}

1. Cetti R, Christensen S, Ejsted R. Operative versus nonoperative treatment of Achilles tendon rupture. Am J Sports Med 1993;21(6):791799. DOI: $10.1177 / 036354659302100606$.

2. Longo UG, Petrillo $S$, Maffulli N, et al. Acute achilles tendon rupture in athletes. Foot Ankle Clin 2013;18(2):319-338. DOI: 10.1016/j. fcl.2013.02.003.

3. Willits $K$, Amendola A, Bryant $D$, et al. Operative versus nonoperative treatment of acute Achilles tendon ruptures: a multicenter randomized trial using accelerated functional rehabilitation. J Bone Joint Surg Am 2010;92(17):2767-2775. DOI: 10.2106/JBJS.I.01401.

4. Wong J, Barrass V, Maffulli N. Quantitative review of operative and nonoperative management of achilles tendon ruptures. Am J Sports Med 2002;30(4):565-575. DOI: 10.1177/03635465020300041701.

5. Khan RJ, Fick D, Keogh A, et al. Treatment of acute achilles tendon ruptures. A meta-analysis of randomized, controlled trials. J Bone Joint Surg Am 2005;87(10):2202-2210. DOI: 10.2106/JBJS.D.03049.

6. Ma GW, Griffith TG. Percutaneous repair of acute closed ruptured Achilles tendon: a new technique. Clin Orthop Relat Res 1977;128(128):247-255. DOI: 10.1097/00003086-197710000-00036.

7. Hockenbury RT, Johns JC. A biomechanical in vitro comparison of open versus percutaneous repair of tendon Achilles. Foot Ankle 1990;11(2):67-72. DOI: 10.1177/107110079001100202.

8. Cretnik A, Zlajpah L, Smrkolj V, et al. The strength of percutaneous methods of repair of the Achilles tendon: a biomechanical study. Med Sci Sports Exerc 2000;32(1):16-20. DOI: 10.1097/00005768-20000100000004.

9. Cretnik A, Kosanović M, Smrkolj V. Percutaneous suturing of the ruptured Achilles tendon under local anesthesia. J Foot Ankle Surg 2004;43(2):72-81. DOI: 10.1053/j.jfas.2004.01.008.

10. Ceccarelli F, Berti L, Giuriati L, et al. Percutaneous and minimally invasive techniques of Achilles tendon repair. Clin Orthop Relat Res 2007:458:188-193. DOI: 10.1097/BLO.0b013e3180396f07.

11. Thompson TC. A test for rupture of the tendo Achillis. Acta Orthop Scand 1962;32(1-4):461-465. DOI: 10.3109/17453676208989608.

12. Citak M, Knobloch K, Albrecht $\mathrm{K}$, et al. Anatomy of the sural nerve in a computer-assisted model: implications for surgical minimal-invasive Achilles tendon repair. Br J Sports Med 2007;41(7):456-458. DOI: 10.1136/bjsm.2006.031328.

13. Maffuli N. Rupture of the Achilles tendon. J Bone Joint Surg 1999;81A(7):1019-1036. DOI: 10.2106/00004623-199907000-00017.

14. Asplund CA, Best TM. Achilles tendon disorders. BMJ 2013;346 (mar12 1):f1262. DOI: 10.1136/bmj.f1262.

15. Ahmed IM, Lagopoulos $M$, McConnell $P$, et al. Blood supply of the Achilles tendon. J Orthop Res 1998;16(5):591-596. DOI: 10.1002/ jor.1100160511.

16. Klein W, Lang DM, Saleh M. The use of the Ma-Griffith technique for percutaneous repair of fresh ruptured tendo Achillis. Chir Organi Mov 1991;76(3):223-228.

17. Compston A. Aids to the investigation of peripheral nerve injuries. Medical Research Council: Nerve Injuries Research Committee. His Majesty's Stationery Office: 1942; p. 48. 